\title{
Feedback learning opportunities from medical student logs of paediatric patients
}

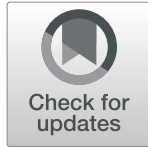

Helen M. Wright ${ }^{1,2,3^{*}}$ D, Moira A. L. Maley ${ }^{1}$, Denese E. Playford ${ }^{1}$, Pam Nicol ${ }^{2}$ and Sharon F. Evans ${ }^{1}$

\begin{abstract}
Background: Feedback can alter medical student logging practices, although most learners feel feedback is inadequate. A varied case mix in rural and urban contexts offers diverse clinical encounters. Logs are an indicator of these clinical experiences, and contain opportunities for feedback, which can greatly influence learning: we labelled these 'feedback learning opportunities' (FLOs).

We asked:

How often do FLOs occur?

What are the case complexities of rural compared to urban paediatric logs?

Do more complex cases result in more FLOs?

Methods: In Western Australia, 25\% of medical students are dispersed in a Rural Clinical School (RCSWA) up to 2175 miles $(3500 \mathrm{~km})$ from the city. Urban students logged 20 written cases; rural students logged a minimum of 25 paediatric cases electronically. These were reviewed to identify FLOs, using a coding convention. FLO categories provided a structure for feedback: medical, professionalism, insufficient, clinical reasoning, student wellbeing, quality and safety, and sociocultural. Each log was assigned an overall primary, secondary or tertiary case complexity.

Results: There were 76 consenting students in each urban and rural group, providing 3034 logs for analysis after exclusions. FLOs occurred in more than half the logs, with significantly more rural (OR 1.35 95\% Cl 1.17, 1.56; $p<0.0001)$. Major FLOs occurred in over a third of logs, but with no significant difference between rural and urban (OR 1.10 $95 \% \mathrm{Cl} 0.94,1.28 ; p=0.24)$. Medical FLOs were the most common, accounting for $64.0 \%$ of rural and $75.2 \%$ of urban FLOs (OR $1.7195 \% \mathrm{Cl} 1.37,2.12 ; p<0.0001$ ). Students logged cases with a variety of complexities. Most cases logged by urban students in a tertiary healthcare setting were of primary and secondary complexity. Major medical FLOs increased with increasing patient complexity, occurring in $32.1 \%$ of tertiary complexity cases logged by urban students $(p<0.001)$.

Conclusions: Case logs are a valuable resource for medical educators to enhance students' learning by providing meaningful feedback. FLOs occurred often, particularly in paediatric cases with multiple medical problems. This study strengthens recommendations for regular review and timely feedback on student logs. We recommend the FLOs categories as a framework for medical educators to identify FLOs.
\end{abstract}

Keywords: Feedback, Logs, Paediatric, Rural

\footnotetext{
* Correspondence: Helen.Wright@rcswa.edu.au

${ }^{1}$ The Rural Clinical School of Western Australia, Faculty of Health and Medical Sciences, University of Western Australia, M706, 35 Stirling Highway, Crawley, WA 6009, Australia

${ }^{2}$ Division of Paediatrics, Faculty of Health and Medical Sciences, University of Western Australia, M501, 35 Stirling Highway, Crawley, WA 6009, Australia Full list of author information is available at the end of the article
}

(c) The Author(s). 2019 Open Access This article is distributed under the terms of the Creative Commons Attribution 4.0 International License (http://creativecommons.org/licenses/by/4.0/), which permits unrestricted use, distribution, and reproduction in any medium, provided you give appropriate credit to the original author(s) and the source, provide a link to the Creative Commons license, and indicate if changes were made. The Creative Commons Public Domain Dedication waiver (http://creativecommons.org/publicdomain/zero/1.0/) applies to the data made available in this article, unless otherwise stated. 


\section{Background}

Feedback on cases that students have encountered is central to, and can greatly influence, learning. Feedback has been defined as information provided about some aspect of the appraisee's performance or understanding [1]. Feedback has been shown to alter medical student logging practices [2], although most learners don't feel they get adequate or effective feedback [3].

We have previously reported alignment of the quantity and variety of paediatric presentations logged by rural and urban medical students with Australian paediatric hospital admissions [4]. Review of student logs during the earlier research highlighted that case logs contain numerous opportunities for medical educators to provide valuable feedback to students. We chose to name these instances 'feedback learning opportunities' (FLOs) for this study. We believe such opportunities constitute a valuable addition to case-based teaching, but the extent to which these FLOs occur is unknown. The present paper provides detail on FLO frequency and severity.

In Australia, as in other countries, medical students are taught in rural and urban settings [5]. These settings provide medical students with exposure to a varied case mix, including different case complexities, essential to support clinical learning $[6,7]$. We wanted to know whether the diverse settings and case complexities reflected differences in FLOs.

Understanding the degree to which FLOs were present in urban vs rural case logs, and how this relates to case complexity, could help guide faculty development for the recognition of FLOs and their use in student education. We therefore designed this study to provide an evidence base about the potential educational value of logs as a resource for medical educators to provide feedback to students.

We asked:

How often do feedback learning opportunities (FLOs) occur in medical student logs?

What are the case complexities of rural compared to urban paediatric logs?

Do more complex cases result in more feedback

learning opportunities (FLOs)?

The results would primarily inform practice in the Australian context, however, as other countries have both rural and urban settings, the findings will have implications for other disciplines, institutions and clinical contexts.

\section{Methods}

\section{Study design}

This study is a retrospective audit of paediatric logs entered by all consenting medical students from an established medical school at the University of Western Australia (UWA). Consent, ethics approval, inclusions, exclusions and log categorisation were detailed in the previous paper [4].

\section{Participants and settings}

In Western Australia, 25\% of medical students are widely dispersed up to 2175 miles $(3500 \mathrm{~km})$ from the university's city campus in a Rural Clinical School (RCSWA), for one academic year. RCSWA sites are all classified as rural or remote [8], with students predominantly community based.

\section{Logging instructions to students}

Urban students were required to log and submit 20 paediatric cases in a written logbook. They were instructed to note clinical problems, history, examination, investigations, working diagnosis and differentials, a problem based management plan and reflection on learning. Specific requirements for cases logged were two outpatients; one Aboriginal child, where it was essential to apply holistic case management; and two developmental assessments.

Rural students were required to $\log$ a minimum of 25 paediatric cases electronically using a custom-built web database. Cases were from the core paediatric presentations in the guidebook. Specific requirements for cases logged were 10\% Aboriginal cases; and 10\% to include personal and professional development topics. Details included patient demographics, where students encountered cases, the clinical problem(s), differential diagnosis and formulation and follow-up. Case logs were to be discussed monthly with a medical educator, with three timetabled reviews during the year to demonstrate adequate numbers and quality of cases.

Satisfactory completion of these logged cases was a barrier assessment for urban and rural students.

\section{Outcomes measured}

To answer the study questions we measured:

Frequency, categories and severity of FLOs in logs of paediatric cases.

Primary, secondary and tertiary levels of complexity of cases logged.

\section{The coding convention}

As an outcome of iterative reading of multiple logs, described in an earlier paper, a coding convention was created by the main author (HW), who reviewed all case logs. The convention is described below, and includes criteria for identifying and categorising different kinds of FLOs. 


\section{Definition of feedback learning opportunities (FLOs)}

Logged cases were reviewed by HW, using the coder's experience as a medical educator, to identify where feedback could be provided to students. Since medical students are known to under-recognise diagnoses [9], they may need specific feedback from teachers to confirm appropriate entries, advise if more information is required, consider all clinical problems for each patient, develop clinical reasoning skills [7], correct inaccuracies [10], restructure understandings, or provide alternatives to learning [1]. Accordingly, FLO categories were developed to assist medical educators with a structure for feedback when reviewing student logs. These were: medical, professionalism, insufficient, clinical reasoning, student wellbeing, quality and safety, and sociocultural.

\section{Case complexity}

Each log was assigned an overall primary, secondary or tertiary case complexity for the whole case by HW. Coding conventions for complexity were that if a logged case needed subspecialist input - eg. diabetes, paediatric surgery, tertiary visiting outpatients then it was coded as tertiary complexity. If the case could have been admitted to a secondary hospital under care of the paediatrics team, or could have been seen in a general paediatric outpatient clinic then it was coded as secondary complexity. If the case could be managed by a GP then it was coded as primary complexity. Newborns were coded as primary complexity if uncomplicated; secondary if involvement of the paediatric team was required; tertiary if neonatal care or transfer was required.

\section{Procedures}

\section{Criteria for each FLO category}

FLO categories developed by HW were labelled as Medical FLOs: where the student should have considered all clinical problems of the case but didn't; as Professionalism FLOs: where logs suggested the student may have benefited from a discussion about professional issues or behaviours with a medical educator; as Insufficient FLOs: where more information was required to interpret the case; as Clinical reasoning FLOs: where there was no working diagnosis, or incomplete differential diagnosis; as Student wellbeing FLOs: where the log raised concerns about student welfare; as Sociocultural FLOs: where students needed to log relevant social history; as Quality and safety FLOs: where cases had a potential adverse outcome, or where inaccuracies should be corrected. Where appropriate, more than one FLO was allocated, for example clinical reasoning and quality and safety where a missing differential diagnosis had the potential for a poor patient outcome.

\section{FLO severity}

Appropriate logs where feedback was not required were coded as no FLO. We used 'could' or 'should' as terms to determine FLO severity, defined as minor or major. FLOs were determined as major if a student should have had recommendations made by a teacher about the content of the case logged, as the FLO was sufficient to warrant feedback. Minor FLOs included simple omissions or lack of information, where a medical educator could have provided some feedback, however this was desirable not a necessity. For example, an infant with a first ear infection where a student could have assessed development would have a minor medical category allocated; an infant with recurrent ear infections and glue ear where a student should have assessed development to look for speech and language delay would have had a major medical category allocated. Further examples and scoring are provided in Appendix 1.

\section{Statistical analysis}

Excel data were imported into SAS (SAS Institute Inc. Cary, NC, USA V9.4). Rural-urban comparisons were made using Chi square and Fisher's exact test for frequency data. The cell $\mathrm{X}^{2}$ test was used for clarification to identify parts of tables where dependencies between row and column categories may exist.

Inter- and intra-rater agreement for patient complexity and major FLOs between HW (previously a rural paediatrician, currently an urban specialist general paediatrician) and an experienced rural general paediatrician was undertaken on a subset of $1 \%$ of case logs.

Urban/rural inter-rater agreement for complexity was measured using a weighted Cohen's Kappa coefficient, as complexity was ordered from primary to secondary to tertiary. Agreement for whether a student had a major FLO was measured using a simple Kappa coefficient.

\section{Results}

Seventy six students gavewritten consent in each of the urban and rural groups (76/107 urban and 76/77 rural). After exclusions (cases seen in rural general practices, adults, antenatal cases and duplicates) there were 1516 urban and 1518 rural paediatric logs for analysis.

\section{How often do FLOs occur?}

FLOs occurred in more than half of cases logged, with significantly more for rural compared to urban students (OR 1.35 95\% CI 1.17, 1.56; $p<0.0001)$. For rural students, 666 (43.9\%) of cases had no FLO, 618 (40.7\%) had one FLO and $234(15.4 \%)$ had more than one FLO; for urban students 778 (51.3\%) had no FLO, $612(40.4 \%)$ had one FLO and $125(8.2 \%)$ had more than one FLO $(p<0.0001)$. Major FLOs occurred in over a third of cases logged, but with no 
significant difference between rural and urban logs (OR 1.10 95\% CI 0.94, 1.28; $p=0.24$ ).

\section{FLO categories for all logs}

Medical FLOs were the most common, accounting for $64.0 \%(545 / 852)$ of all rural and $75.2 \%(555 / 738)$ of all urban FLOs (OR 1.71 95\%CI 1.37, 2.12; $p<0.0001$ ). Rural students had significantly more FLOs relating to professionalism issues $(14.6 \%$ v $7.1 \%$, OR $2.2395 \%$ CI $1.75,2.84 ; p<0.001)$; insufficient clinical information (8.6\% v $1.5 \%$, OR $6.3695 \%$ CI 4.02, 10.05, $p<0.001)$; relating to clinical reasoning ( $5.4 \%$ v $3.8 \%$, OR $1.4695 \%$ CI $1.03,2.07 ; p=0.03$ ); and regarding student wellbeing $(0.5 \% \mathrm{v}$ none $p=0.016)$. Urban students had significantly more quality and safety FLOs than rural students (4.8\% v $2.2 \%$, OR $2.2895 \%$ CI 1.50, 3.46; $p<0.001$ ).

\section{What were the case complexities of rural compared to urban paediatric logs?}

Students logged paediatric cases with a variety of overall clinical complexities across all healthcare settings. Most cases logged by urban students in a tertiary healthcare setting were of primary and secondary complexity. Although the distribution patterns of case complexity appeared essentially the same, urban students logged significantly more cases of tertiary complexity than rural students (OR $1.3095 \%$ CI 1.12, 1.51; $p=0.0018$ ), shown in Table 1.

Case complexity for cases logged in emergency departments was the same regardless of the rural or urban setting, with more than a third of primary complexity. However, the patterns of inpatient and outpatient logs were different between urban and rural students, when analysed specifically. More than half the cases rated as tertiary complexity cases logged by urban students were inpatients. In contrast, more than a third of these highly complex cases were seen as outpatients by rural students. More than half the rural inpatient logs were of secondary level complexity, with significantly more primary, and fewer tertiary complexity inpatients than urban cases logged $(p<0.0001)$. Urban students logged significantly fewer outpatients of secondary complexity compared to rural.

\section{Did more complex cases result in more major FLOs?}

Table 2 demonstrates the pattern of major FLOs by case complexity. Major medical FLOs increased with increasing patient complexity, particularly for urban students where one third of tertiary complexity logs had a major medical FLO $(p<0.001)$, with one fifth for rural students (OR $1.9595 \%$ CI 1.47, 2.59). None of the other major FLO categories increased with increasing complexity.

More major FLOs occurred in rural primary complexity cases logged compared to urban (OR $1.5495 \% \mathrm{CI}$ $1.06,2.23 ; p=0.025)$. There was no difference for secondary complexity cases (OR $0.9395 \%$ CI 0.74, 1.17; $p=$ $0.60)$. Consequently, fewer tertiary complexity cases logged by rural students had some form of FLO (OR 0.75 95\% CI 0.59, 0.96; $p=0.021$ ).

Inter-rater agreement for patient complexity with a rural general paediatrician was 0.95 (95\% CI $0.86,1.00)$ and for major FLO was 0.94 (CI 0.82, 1.00). Similarly, intra-rater agreements were $0.87(0.73,1.00)$ and 0.88 $(0.72,1.00)$ respectively.

\section{Discussion}

Case logs are a valuable resource for medical educators to enhance students' learning experiences by providing meaningful feedback. Feedback learning opportunities (FLOs) occurred often in medical student logs of paediatric patients, particularly in cases with multiple medical problems. This study strengthens recommendations for regular review [11] and timely feedback on student logs [12].

FLOs occurred often, and were mostly medical, fitting with previous research that students under-report

Table 1 Case complexity across healthcare settings for urban and rural case logs

\begin{tabular}{|c|c|c|c|c|c|c|c|c|}
\hline \multirow[b]{3}{*}{ Location } & \multicolumn{6}{|c|}{ Case Clinical Complexity } & \multirow{2}{*}{\multicolumn{2}{|c|}{$\begin{array}{l}\text { Total } \\
\%\end{array}$}} \\
\hline & \multicolumn{2}{|l|}{ Primary } & \multicolumn{2}{|c|}{ Secondary } & \multicolumn{2}{|c|}{ Tertiary } & & \\
\hline & Rural & Urban & Rural & Urban & Rural & Urban & Rural & Urban \\
\hline $\begin{array}{l}\text { Emergency } \\
\%\end{array}$ & $\begin{array}{l}164 \\
35.8 \%\end{array}$ & $\begin{array}{l}237 \\
33.5 \%\end{array}$ & $\begin{array}{l}202 \\
44.1 \%\end{array}$ & $\begin{array}{l}294 \\
41.6 \%\end{array}$ & $\begin{array}{l}92 \\
20.1 \%\end{array}$ & $\begin{array}{l}176 \\
24.9 \%\end{array}$ & $\begin{array}{l}458 \\
30.2 \%\end{array}$ & $\begin{array}{l}707 \\
46.6 \%\end{array}$ \\
\hline $\begin{array}{l}\text { Inpatient } \\
\%\end{array}$ & $\begin{array}{l}117 \\
16.9 \%\end{array}$ & $\begin{array}{l}14 \\
2.3 \%\end{array}$ & $\begin{array}{l}353 \\
50.9 \%\end{array}$ & $\begin{array}{l}285 \\
47.0 \%\end{array}$ & $\begin{array}{l}223 \\
32.2 \%\end{array}$ & $\begin{array}{l}307 \\
50.7 \%\end{array}$ & $\begin{array}{l}693 \\
45.7 \%\end{array}$ & $\begin{array}{l}606 \\
40.0 \%\end{array}$ \\
\hline $\begin{array}{l}\text { Outpatient } \\
\%\end{array}$ & $\begin{array}{l}22 \\
6.8 \%\end{array}$ & $\begin{array}{l}17 \\
11.2 \%\end{array}$ & $\begin{array}{l}131 \\
40.7 \%\end{array}$ & $\begin{array}{l}41 \\
27.0 \%\end{array}$ & $\begin{array}{l}169 \\
52.4 \%\end{array}$ & $\begin{array}{l}94 \\
61.8 \%\end{array}$ & $\begin{array}{l}322 \\
21.1 \%\end{array}$ & $\begin{array}{l}152 \\
10.0 \%\end{array}$ \\
\hline $\begin{array}{l}\text { Other } \\
\%\end{array}$ & $\begin{array}{l}13 \\
28.9 \%\end{array}$ & $\begin{array}{l}5 \\
9.8 \%\end{array}$ & $\begin{array}{l}18 \\
40.0 \%\end{array}$ & $\begin{array}{l}34 \\
66.7 \%\end{array}$ & $\begin{array}{l}14 \\
31.1 \%\end{array}$ & $\begin{array}{l}12 \\
23.5 \%\end{array}$ & $\begin{array}{l}45 \\
3.0 \%\end{array}$ & $\begin{array}{l}51 \\
3.4 \%\end{array}$ \\
\hline Total & 316 & 273 & 704 & 654 & 498 & 589 & 1518 & 1516 \\
\hline$\%$ of cases & $20.8 \%$ & $18.0 \%$ & $46.4 \%$ & $43.1 \%$ & $32.8 \%$ & $38.9 \%$ & & \\
\hline
\end{tabular}

other included school, home, overseas electives 
Table 2 Major feedback learning opportunities (FLOs) by complexity

\begin{tabular}{|c|c|c|c|c|c|c|c|c|}
\hline \multirow[b]{3}{*}{ Major FLO } & \multicolumn{8}{|c|}{ Case Clinical Complexity } \\
\hline & \multicolumn{2}{|l|}{ Primary (\%) } & \multicolumn{2}{|c|}{ Secondary (\%) } & \multicolumn{2}{|l|}{ Tertiary (\%) } & \multicolumn{2}{|l|}{ Total } \\
\hline & Rural & Urban & Rural & Urban & Rural & Urban & Rural & Urban \\
\hline Any Major FLO & 97 (30.7\%) & $61(22.3 \%)$ & $220(31.3 \%)$ & $214(32.7 \%)$ & $178(35.7 \%)$ & $251(42.6 \%)$ & $495(32.6 \%)$ & $526(34.7 \%)$ \\
\hline Medical & $30(9.5 \%)$ & $30(11.0 \%)$ & $115(16.3 \%)$ & $138(21.1 \%)$ & 97 (19.5\%) & $189(32.1 \%)$ & 242 (15.9\%) & $357(23.5 \%)$ \\
\hline Professionalism & 49 (15.5\%) & 19 (7.0\%) & $69(9.8 \%)$ & $37(5.7 \%)$ & $63(12.7 \%)$ & $43(7.3 \%)$ & $181(11.9 \%)$ & 99 (6.5\%) \\
\hline Clinical reasoning & $15(4.7 \%)$ & $9(3.3 \%)$ & $41(5.8 \%)$ & $22(3.4 \%)$ & $22(4.4 \%)$ & $17(2.9 \%)$ & $78(5.1 \%)$ & 48 (3.2\%) \\
\hline Insufficient & $20(6.3 \%)$ & $2(0.7 \%)$ & $65(9.2 \%)$ & $11(1.7 \%)$ & $36(7.2 \%)$ & $7(1.2 \%)$ & $121(8.0 \%)$ & $20(1.3 \%)$ \\
\hline Social & $2(0.6 \%)$ & $3(1.1 \%)$ & $6(0.9 \%)$ & $10(1.5 \%)$ & $2(0.4 \%)$ & $15(2.5 \%)$ & $10(0.7 \%)$ & $28(1.8 \%)$ \\
\hline Quality and Safety & $9(2.8 \%)$ & $13(4.8 \%)$ & $14(2.0 \%)$ & $34(5.2 \%)$ & $6(1.2 \%)$ & $23(3.9 \%)$ & $29(1.9 \%)$ & $70(4.6 \%)$ \\
\hline Wellbeing & $0(0 \%)$ & $0(0 \%)$ & $1(0.1 \%)$ & $0(0 \%)$ & $6(1.2 \%)$ & $0(0 \%)$ & 7 (0.5\%) & $0(0 \%)$ \\
\hline Total cases & 316 & 273 & 704 & 654 & 498 & 589 & 1518 & 1516 \\
\hline
\end{tabular}

medical conditions $[9,13]$. We acknowledge that cases with any FLO may have benefited from review with a medical educator, however the feedback categories suggested in this paper present a framework for discussions. They provided information about a students' understanding and were specific, addressed inaccuracies, identified where students may have needed advice, reinterpretation, and/or alternatives provided to their learning, as recommended in the feedback literature [1].

Rural students had more FLOs overall, however there was no difference in major FLOs between rural and urban students. The increased FLOs identified for rural students were minor, which may be explained by increased insufficient and professionalism FLOs by rural students. This has been addressed by amending logging requirements for rural students following this study, and are unlikely to have future implications.

This study demonstrates that urban and rural clinical schools in WA provide medical students with a variety of patient complexities. Students logged mostly primary and secondary complexity cases, in keeping with the curriculum learning outcomes for urban [14] and rural medical graduates [15].

Emergency departments (EDs) were frequently used by rural and urban students to log paediatric cases, with the same proportions of case complexities. Students logged many primary complexity cases logged in EDs, consistent with $15.4 \%$ of paediatric presentations to Australian EDs allocated Category 5, or "low urgency" using the Australasian Triage Scale [16].

Rural outpatient clinics, including visiting specialist clinics by paediatricians and subspecialists [17], were a valuable resource for rural students to log secondary and tertiary complexity paediatric cases [18]. Urban students underutilised outpatients, particularly secondary complexity cases.

Varying referral and admission patterns for rural patients in Australia [19, 20], may explain the different patterns of inpatient and outpatient logs between urban and rural students. These are relevant to rural medical students' learning as the higher proportion of rural primary complexity inpatients reflects the health needs of the rural community.

A substantial proportion of tertiary complexity cases logged had a major medical FLO, which may relate to higher acuity patients, as most logs were of tertiary paediatric hospital inpatients. Feedback on paediatric patients with multiple medical problems is therefore recommended.

\section{Limitations}

The contrasting logging methods used by rural and urban students could explain some of the differences observed in this study. Although students using electronic logs recorded more patient problems in one study [21], the overall influence of written or electronic logs on the accuracy of clinical information recorded is unclear [22].

Logs reflect one aspect of students' actual learning experiences, so FLOs may have been overestimated as logs document only what the student has recorded. It is also possible students had received feedback from medical educators, or counselling, about confronting clinical scenarios logged.

Students' own style and degree of student supervision are variables which influence student learning [6]. Awareness of the students' learning approach (deep or surface) may have been useful as a surface learning approach may underlie some of the insufficient FLOs.

Analysing logs for FLOs assumes all students were given equivalent instruction on how to $\log$ using best practice by medical educators in urban and rural sites. The increased professionalism FLOs by rural students constituted only a small proportion of FLOs, so is noteworthy but not of concern, and may be related to the requirement for rural students to $\log 10 \%$ of cases relating to personal and professional development. 
This study was not designed to determine if students chose to strategically log less complex patients. However, the finding that students logged mostly primary and secondary cases fits with clinical practice, so student choice was unlikely to have highly influenced the complexity of cases logged.

Interpretation of the logs as entered by the student was subjective, however the same criteria were used for urban and rural. The potential for bias was minimised, as HW has experience in urban and rural schools, and clinical settings.

Ideally, review of the patients' medical records in parallel with the students' logs would have provided more comprehensive clinical information, perhaps increasing the quantity of medical FLOs, and strengthening the recommendations for feedback.

As logs were de-identified this was not possible. However, the large number of logged cases analysed in this study allowed a greater breadth of FLO type and grade to be compared.

\section{Practical implications}

The data presented in this paper suggests most logs of paediatric patients by rural and urban medical students in Western Australia contain opportunities for medical educators to provide them with feedback that may enhance their learning. Next steps are to ensure medical educators can recognise FLO's in medical student logs and know how to give effective feedback to students once identified.

We acknowledge the detail undertaken in this study by the principal investigator goes beyond the scope of most marking processes, and the coding convention would need further testing before widespread use. Nonetheless, we anticipate most FLOs would be easily identified by medical educators.

We recommend using the FLOs categories of medical, professionalism, insufficient, clinical reasoning, student wellbeing, quality and safety, and sociocultural as a practical framework for medical educators to help identify FLOs and aid discussions.

Medical FLOs discussions could guide students to consider all aspects of the case [9] such as co-existing diagnoses, developmental assessment or adolescent health issues. Professionalism FLOs could stimulate conversations encouraging reflection, suggest use of medical terminology, and debating ethical issues to aid personal and professional development [23]. Clinical reasoning FLO discussions could assist students with diagnostic skills [7]. Insufficient FLOs could stimulate conversations about ensuring relevant details are included. Sociocultural FLOs may result in discussions about cigarette smoking, the impact of living in a remote area, or relevant cultural issues. Quality and safety FLOs may emphasise the importance of patient safety in medical student education [24]. Wellbeing FLOs may identify students who should meet a medical educator for academic or personal support, if required [22].

This study examined logs from one clinical discipline and FLOs may occur with similar frequency in other disciplines and Schools. Further research using the methods described, after refining and testing the coding convention, could be undertaken to confirm this.

\section{Conclusions}

Student logs of paediatric patients contain many feedback learning opportunities, so timely review and feedback is strongly recommended for their educational benefit to be realised. Student logs evidence clinical encounters but more importantly, students' current thinking. Logs can provide teachers with opportunities to have meaningful conversations with students during feedback sessions. These data indicate that there is an educational requirement for medical educators to review cases logged to enhance the learning experience.

\section{Appendix}

Feedback learning opportunities (FLO) scoring, and examples

TFOs were scored from 0 to 5 ( 0 - none, 1 - one minor, 2 multiple minor, 3 one major, 4 more than one major and 5 incorrect/dangerous information in the student $\log$.

\section{Medical}

"Could have reviewed tracheo-oesophageal fistula and potential complications."

"Could have reviewed allergic rhinitis and ADHD."

"Should have explored eczema."

"Stridor not explored."

\section{Professionalism}

"Review of this log book an opportunity to improve written communication."

"..concerning terminology that could be addressed by review of their logging."

\section{Insufficient}

"Should have more detail."

"Minimal info in case."

"Insufficient examination."

\section{Clinical reasoning}

"Incorrect diagnosis concern."

"Needed to include pertussis in differential."

\section{Wellbeing}

"Student needs debrief post child sexual assault case."

"Student needs debrief." - post stillbirth. 


\section{Quality and safety}

"Unsafe to give trimeprazine to under 2 year old."

\section{Socio-cultural}

"Opportunity to reflect on issues for Aboriginal child from rural community."

"Should have smoking history in child with multiple asthma presentations."

"Social history required for recurrent ear disease."

"Should have identified need to use an interpreter."

\section{Abbreviations}

AlHW: Australian Institute of Health and Welfare; FLOs: Feedback learning opportunities; MDCs: Major diagnostic categories; RCSWA: Rural Clinical School of Western Australia; UWA: University of Western Australia

\section{Acknowledgements}

We gratefully acknowledge the professional work of Malcolm Evans as the database architect and programmer for the web-based logging resource "Student eLog" used by RCSWA students to capture their clinical experience during their LIC (www.surak.com.au); Michael Clark Research Assistant for data entry; and Sharon Peters for critical review.

\section{Funding}

HW received an early career research grant of Au\$30,000 from the University of Western Australia. This was used to employ a research assistant to set up the database.

\section{Availability of data and materials}

The datasets used and/or analysed during the current study are available from the corresponding author on reasonable request.

\section{Authors' contributions}

HW was responsible for study concept. Study design was by HW, MM, DP, PN and SE. HW was the main contributor to acquisition of data. SE undertook data analysis. HW, MM and SE were involved in interpretation of data. HW, MM, DP, PN and SE have all been involved in drafting the manuscript and revising it critically for important intellectual content. HW, MM, DP, PN and SE have given final approval of the version to be published; have participated sufficiently in the work to take public responsibility for appropriate portions of the content; and have agreed to be accountable for all aspects of the work in ensuring that questions related to the accuracy or integrity of any part of the work are appropriately investigated and resolved.

\section{Authors' information}

Helen Wright is a General Paediatrician in the Child and Adolescent Health Service in Perth. She is Senior Lecturer in the Rural Clinical School of Western Australia and the Division of Paediatrics, School of Medicine, Faculty of Health and Medical Sciences, University of Western Australia. She is Co-lead of the WA Child and Youth Health Network.

Moira Maley is a senior medical education technology academic with a special interest in experiential and transformative learning in a range of contexts including rural medical education.

Sharon Evans is a biostatistician with over 40 years' experience in many aspects of medicine and medical education. She has extensive experience in paediatrics and obstetrics research in England, New Zealand and Australia. Pam Nicol is a nurse and medical educator, who worked for many years in the School of Paediatrics and Child Health.

Denese Playford is the academic coordinator for The Rural Clinical School of Western Australia, working collaboratively with colleagues across the State.

\section{Ethics approval and consent to participate}

Ethics approval was obtained from the University of Western Australia Ethics Committee approval RA/4/1/5497. Consent to participate and for publication was obtained from students.

\section{Consent for publication}

Not applicable.

\section{Competing interests}

The authors declare that they have no competing interests.

\section{Publisher's Note}

Springer Nature remains neutral with regard to jurisdictional claims in published maps and institutional affiliations.

\section{Author details}

${ }^{1}$ The Rural Clinical School of Western Australia, Faculty of Health and Medical Sciences, University of Western Australia, M706, 35 Stirling Highway, Crawley, WA 6009, Australia. ${ }^{2}$ Division of Paediatrics, Faculty of Health and Medical Sciences, University of Western Australia, M501, 35 Stirling Highway, Crawley, WA 6009, Australia. 'Department of General Paediatrics, Perth Children's Hospital, 15 Hospital Ave, Nedlands, WA 6009, Australia.

Received: 23 August 2018 Accepted: 25 March 2019

Published online: 11 April 2019

\section{References}

1. Hattie J, Timperley H. The power of feedback. Rev Educ Res. 2007;77(1):81-112.

2. Delzell JE Jr, Chumley HS, Clarkson AL. Mid-clerkship feedback is effective in changing students' recorded patient encounters. Fam Med. 2011;43(8):586-8.

3. Ramani S, Krackov SK. Twelve tips for giving feedback effectively in the clinical environment. Med Teach. 2012;34(10):787-91.

4. Wright HM, Maley MAL, Playford DE, Nicol P, Evans SF. Paediatric case mix in a rural clinical school is relevant to future practice. BMC Med Educ. 2017; 17(1):237.

5. Maley M, Worley P, Dent J. Using rural and remote settings in the undergraduate medical curriculum: AMEE guide no. 47. Med Teach. 2009; 31(11):969-83.

6. de Jong J, Visser M, Van Dijk N, van der Vleuten C, Wieringa-de Waard M. A systematic review of the relationship between patient mix and learning in work-based clinical settings. A BEME systematic review: BEME guide no. 24. Med Teach. 2013:35(6):e1181-96.

7. Bowen JL. Educational strategies to promote clinical diagnostic reasoning. $\mathrm{N}$ Engl J Med. 2006:355(21):2217-25.

8. Australian Bureau of Statistics. Australian Statistical Geography Standard (ASGS): Volume 5 - Remoteness Structure 1270.0.55.005 [Available from: http://www.abs.gov.au. Accessed 29 Aug 2016.

9. Raghoebar-Krieger HM, Sleijfer D, Bender W, Stewart RE, Popping R. The reliability of logbook data of medical students: an estimation of interobserver agreement, sensitivity and specificity. Med Educ. 2001;35(7): 624-31.

10. Ericsson KA. Deliberate practice and the acquisition and maintenance of expert performance in medicine and related domains. Acad Med. 2004; 79(10):S70-81.

11. Schuttpelz-Brauns K, Narciss E, Schneyinck C, Bohme K, Brustle P, MauHolzmann $U$, et al. Twelve tips for successfully implementing logbooks in clinical training. Med Teach. 2016;38(6):564-9.

12. Li ST, Smith S, Gigante J. A national survey of pediatric clerkship directors' approaches to meeting the LCME ED-2 requirement for quantified patient criteria for medical students. Teach Learn Med. 2007:19(4):352-6.

13. Denton GD, Hoang T, Prince L, Moores L, Durning S. Accuracy of medical student electronic logbook problem list entry. Teach Learn Med. 2007;19(4): 347-51

14. Nicol P, Wright H, Panotidis N, Payne D. Paediatrics 5551/5552 unit guidebook. School of Paediatrics and Child Health: University of Western Australia; 2011.

15. Wright H P, S Burgner D, Richmond P, Nicol P. Rural Clinical Practice, RMED5571/5572 and 5573/5574 Paediatrics Guidebook. 2011.2.

16. Acworth J, Babl F, Borland M, Ngo P, Krieser D, Schutz J, et al. Patterns of presentation to the Australian and New Zealand Paediatric emergency research network. Emerg Med Australas. 2009;21(1):59-66.

17. Rural Health West. Specialist Services in Rural Western Australia Gap and Equity Analysis Update. Perth: Rural Health West; 2016. Available at http:// www.ruralhealthwest.com.au.

18. Hiscock H, Roberts G, Efron D, Sewell JR, Bryson HE, Price AM, et al. Children attending Paediatricians study: a national prospective audit of outpatient practice from the Australian Paediatric research network. Med J Aust. 2011; 194(8):392-7. 
19. Britt H, Miller G. C, Valenti L. It's different in the bush: a comparison of general practice activity in metropolitan and rural areas of Australia 19982000. Australian Institute of Health and Welfare. 2001;General practice series, 1442-3022; no. 6

20. Knox S BH, Pan Y, et al. Locality matters: the influence of geography on general practice activity in Australia 1998-2004. . AlHW, Canberra. 2005; General Practice Series No. 17.

21. Sumner $\mathbf{W} 2$ nd. Student documentation of multiple diagnoses in family practice patients using a handheld student encounter log. Proc AMIA Symp. 2001;1067-5027/01:687-90.

22. Denton GD, DeMott C, Pangaro LN, Hemmer PA. Narrative review: use of student-generated logbooks in undergraduate medical education. Teach Learn Med. 2006;18(2):153-64.

23. Gordon J. Fostering students' personal and professional development in medicine: a new framework for PPD. Med Educ. 2003:37(4):341-9.

24. World Health Organization. WHO patient safety curriculum guide for medical schools. Geneva: World Health Organization; 2009. http://www.who. int/iris/handle/10665/44091.

Ready to submit your research? Choose BMC and benefit from:

- fast, convenient online submission

- thorough peer review by experienced researchers in your field

- rapid publication on acceptance

- support for research data, including large and complex data types

- gold Open Access which fosters wider collaboration and increased citations

- maximum visibility for your research: over $100 \mathrm{M}$ website views per year

At $\mathrm{BMC}$, research is always in progress.

Learn more biomedcentral.com/submissions 